\title{
Puericultura y lactancia en Buenos Aires (1934-1943)*
}

\section{Childcare and Breastfeeding in Buenos Aires (1934-1943)}

\author{
Mariana Ángela Dovio** \\ (D) https://orcid.org/0000-0001-9209-1568 \\ Instituto Patagónico de Estudios de Humanidades y Ciencias Sociales \\ Consejo Nacional de Investigaciones Científicas \\ Universidad Nacional del Comahue, Argentina \\ marianadovio@yahoo.com.ar
}

Resumen: El objetivo de este trabajo es estudiar la puericultura y la lactancia desde la publicación Anales de la Sociedad de Puericultura de Buenos Aires, entre 1934 y 1943. Esta Sociedad fue creada en el ámbito de la Dirección Municipal de Protección a la Primera Infancia, donde funcionaron dispensarios de lactantes e institutos de puericultura. El principal hallazgo es que desde los discursos de los Anales se prestó especial interés a la nutrición del lactante como una cuestión científica de la que debía tener conocimiento el puericultor para llevar a cabo su tarea. Además, se desplegaron tecnologías de gobierno en

* Esta investigación se enmarca en el proyecto Tecnologías de Gobierno en Relación a la Peligrosidad Social desde Espacios de Producción Científicos (Buenos Aires, 1933-1950), financiado por el Consejo Nacional de Investigaciones Científicas y Técnicas (CONICET), y en el cual Dovio participó como investigadora asistente.

** Doctora en Ciencias Sociales por la Universidad Nacional de Buenos Aires. Investigadora asistente del Instituto Patagónico de Estudios de Humanidades y Ciencias Sociales-Consejo Nacional de Investigaciones Científicas, Universidad Nacional del Comahue (IPEHCS-CoNICET-UNCO). Línea de investigación: sociología del control social.

cómo citar: Dovio, M. Á. (2022). Puericultura y lactancia en Buenos Aires (1934-1943). Secuencia (112), e1886. DOI: https://doi.org/10.18234/secuencia.v0i112.1886

cc $(1)$ Esta obra está protegida bajo una Licencia Creative Commons Atribución-NoComercial 4.0 Internacional. 
torno a la lactancia orientadas a la prevención y conservación de la salud de los niños y niñas, así como para guiar el comportamiento de las madres. Esta investigación abre el camino para futuras indagaciones sobre la asistencia a la primera infancia y su relación particular con la nutrición.

Palabras clave: puericultura; prevención; dispensarios de lactantes; tecnologías de gobierno; lactancia.

Abstract: This paper seeks to study childcare and breastfeeding in the Annals of the Buenos Aires Childcare Society between 1934 and 1943. This society was created within the sphere of the Municipal Directorate for the Protection of Early Childhood, in which infant dispensaries and Childcare Institutes operated. The main finding is that the discourse in the Annals shows that special interest was given to infant nutrition as a scientific issue of which childcare providers should be aware to perform their task. In addition, government technologies were used in connection with breastfeeding, to achieve health prevention and conservation for children, as well as to guide the behavior of mothers. This research paves the way for future inquiries into early childhood care and its relationship to nutrition.

Keywords: childcare; prevention; infant dispensaries; government technologies; breastfeeding.

Recibido: 30 de julio de 2020 Aceptado: 21 de diciembre de 2020

Publicado: 28 de enero de 2022

\section{INTRODUCCIÓN}

Dara este trabajo nos situamos dentro de la historia de la salud y la infanLia y en la construcción de diálogos en la intersección de ambos campos de análisis. Desde la bibliografía especializada se ha identificado que hacia 1930, en Buenos Aires, hubo una preocupación desde el campo médico por el futuro de la población debido a la disminución de las tasas de inmigración (producto de la crisis económica mundial) y de la natalidad. Al mismo 
tiempo, ocurrió un avance en la institucionalización estatal en la atención de la salud materno-infantil (Biernat y Ramacciotti, 2008; Nari, 2005). En la ciudad de Buenos Aires se consolidó un sistema institucional de protección a la primera infancia y para 1930, dentro de la órbita de la Dirección Municipal de Protección a la Primera Infancia de Buenos Aires, funcionaron 20 dispensarios de lactantes y cinco institutos de puericultura a los que asistían, en mayor parte, mujeres de pocos recursos económicos para recibir leche artificial gratuita para sus hijos, tratamientos y consultas médicas, entre otros servicios. Esto implicó una intervención profesional del médico, respecto a las madres, en relación con la enseñanza de técnicas de higiene y alimentarias. En términos más generales, fue parte de un creciente interés del Estado por la vida privada de los sectores más pobres, en los que se combinó preocupación social con autoritarismo (Di Liscia, 2005, p. 113). También hubo una progresiva profesionalización de disciplinas consideradas auxiliares como enfermería, obstetricia y visitadoras sociales (Gavrila, 2018).

En este contexto se ubicó el desarrollo de los estudios sobre puericultura, tema que ha sido poco explorado en la bibliografía especializada local con algunas excepciones de los últimos años. Este es el caso de María José Billorou (2006), quien ha estudiado la puericultura en la relación de los médicos con la población desde dispensarios de lactantes e institutos de puericultura, estableciendo que la atención de las madres e hijos de los sectores más pobres se sustentó en una higiene positiva. Siguiendo a otros autores, de esta manera se aspiró a la perfectibilidad moral en un momento en que los ciclos epidémicos estaban controlados y surgieron proyectos centrados en enfermedades crónicas, entre ellas, las relacionadas con la alimentación (Armus y Belmartino, 2001; Buschini, 2013). A partir de esta perspectiva, la salud se relacionó con la obtención de un máximo rendimiento físico y laboral. Las madres se convirtieron en destinatarias privilegiadas de las iniciativas generadas para lograr el cuidado del niño sano, en consonancia con la centralidad de la función materna, proceso que, a su vez, fortaleció la necesidad de una cultura de origen científico para la crianza de los niños y niñas (Colángelo, 2013).

Por otro lado, Ana María Briolotti (2016a) ha indagado las relaciones establecidas entre la medicina infantil y los saberes psicológicos, estableciendo que la puericultura se nutrió de la psicología del desarrollo para orientar a los padres en la educación moral y espiritual de los hijos. En especial, el uso médico de las técnicas de evaluación psicológica en dispensarios de lactantes. Roman (2013) ha puesto interés en los aspectos pedagógicos de los 
Manuales de Puericultura de la ciudad de Santa Fe, los cuales tuvieron como objetivo instruir al público femenino en los cuidados de la infancia. Desde la perspectiva de los estudios eugenésicos, Marisa Miranda (2019) ha estudiado el Libro de las madres, escrito por Gregorio Aráoz Alfaro, el cual forma parte de los desarrollos de la puericultura en Argentina.

Retomando estos avances, una de las áreas de vacancia en los estudios sobre la puericultura en Buenos Aires está integrada por el estudio de Anales de la Sociedad de Puericultura de Buenos Aires, producción académica en la que escribieron médicos sobre temas de asistencia a la primera infancia desde dispensarios de lactantes e institutos de puericultura, aportando sus observaciones, relevamientos, propuestas de mejoramiento de atención, entre otros temas que pusieron especial interés en la calidad de la alimentación de los lactantes.

El objetivo de este trabajo es estudiar desde los Anales la puericultura y, en particular, la lactancia, sobre la cual se ensayaron tecnologías de gobierno. Las tecnologías, en general, forman parte de una racionalidad práctica por la que se busca actuar sobre los individuos para que dirijan sus conductas en direcciones determinadas en sentidos coercitivos y también productivos. Implica el ensamblamiento, la vinculación entre fuerzas, artes, destrezas y técnicas que hacen pensable un modo de existencia (Rose, 1990, p. 5). Las tecnologías de gobierno en relación con la lactancia en los Anales implicaron diversas acciones de los médicos, como la vigilancia dietética, la educación y orientación a las madres, acercamiento a centros de salud, formas de asegurar la buena calidad de leches alternativas, entre otras. A la vez, produjeron saberes científicos sobre estos temas. La nutrición del lactante fue considerada una cuestión de la que el puericultor debía tener conocimiento para llevar a cabo su tarea. Respecto a un campo particular como la lactancia, el gobierno supuso la implementación de medidas al respecto de parte de los médicos, construcción de subjetividades deseables y su instauración como problema, esto es, que resultara constituida de cierta manera y caracterizada con elementos singulares.

El corpus documental de esta investigación está integrado por artículos, noticias y notas publicadas en Anales entre 1935 y 1943, año este último en que cambió de nombre a Revista de la Sociedad de Puericultura. Para ello utilizamos una metodología cualitativa de análisis del discurso, entendiendo al último no como una mera relación lingüística, sino como un entramado de enunciados que circulan entre los cuerpos en prácticas que son siempre relaciones de poder que constituyen sentido. La constitución de sentido brota no 
sólo de qué dice sino de quién, a quién, dónde, desde qué posición de poder y en qué entramado se dice (Murillo, 2008). El discurso, entonces, configura una práctica social sujeta a reglas a través de las cuales se forma y se transforma. En tanto espacio de posiciones y de funcionamientos diferenciados, los sujetos tienen un lugar y una función que es desplazable y mutable. La propuesta estriba en el análisis del discurso en función de los lugares que ocupan los sujetos discurrentes en los campos prácticos en los que se despliegan (Foucault, 2005).

Enseguida, presentamos la noción de puericultura, las funciones de dispensarios de lactantes desde principios del siglo xx en Buenos Aires, la Dirección Municipal de Protección a la Primera Infancia y la Dirección de Maternidad e Infancia. Después, describimos la fundación de la Sociedad de Puericultura de Buenos Aires, sus miembros, objetivos y Anales. Luego, nos dedicamos a estudiar discursos y prácticas en torno a la lactancia, tomando en consideración que este fue un eje principal en las acciones de asistencia registradas por los médicos en las agencias estatales estudiadas. Por último, damos cuenta de propuestas de mejoramiento de la atención en dispensarios, en los cuales se combinó la preocupación social con el autoritarismo.

\section{PUERICULTURA. DISPENSARIOS DE LACTANTES Y DIRECCIÓN MUNICIPAL DE PROTECCIÓN A LA PRIMERA INFANCIA. DIRECCIÓN DE MATERNIDAD E INFANCIA EN BUENOS AIRES}

La puericultura puede ser considerada un campo de conocimiento y una política pública que buscó la protección integral de la madre y el niño y se dedicó a diversos aspectos del arte de la crianza. Surgió en el siglo xIx, en Francia, ante la caída de la natalidad, a la par del creciente prestigio médico (Billorou, 2006). Fue popularizada a comienzos del siglo xx por Adolphe Pinard, quien fue uno de los maestros de la obstetricia francesa, fundador de la Sociedad Francesa de Eugenesia y de la Escuela de Puericultura de París. Abogó por políticas pronatalistas y presentó en su país, en 1920, un proyecto para el examen médico prenupcial obligatorio. Además, amplió el campo de la puericultura, para que no sólo abarcara el arte de criar, sino de procrear a los niños en las mejores condiciones posibles (Briolotti, 2018, p. 182). Por otra parte, desde una perspectiva sociológica, Luc Boltanski (1969) estableció que la puericultura se desplegó, 
desde fines del siglo XIX, en los términos de un amplio proyecto pedagógico con fines relativos a implementar la creencia de que se debía reglamentar la vida privada desde la ciencia, a través de consejos dirigidos a las madres, con diferencias en función de los sectores sociales a los que estuvieran dirigidos.

La puericultura tuvo una rápida recepción en Argentina entre los médicos Emilio Coni, Ernesto Gaing y Gregorio Aráoz Alfaro, entre otros (Miranda 2019, p. 159). Este último fue reconocido en el ámbito de la medicina infantil, es autor de El libro de las madres, presidió el Departamento Nacional de Higiene, la Academia Nacional de Medicina y el Instituto Interamericano de Protección a la Infancia. Como ha señalado Miranda (2019), participó en la promoción de la puericultura y la pediatría desde una perspectiva eugenésica, profundizando aspectos relativos a "la selección matrimonial e inmigratoria y procreación intervenida, en cuanto la madre era considerada no tanto por sí misma sino por su carácter de proveedora de vida y salud al niño, el futuro de la patria" (p. 169). Otra de sus preocupaciones fue la nutrición de los niños, por lo que impulsó la creación de la Comisión Nacional de Estudio e Higiene de la Leche, siendo un vocero a favor de la regulación y comercialización de la leche de consumo en Buenos Aires (Buschini, 2018, p. 48).

En el ámbito local, la puericultura tuvo un perfil eminentemente preventivo y extendido a etapas previas al nacimiento, de acuerdo con la concepción de Pinard (Briolotti, 2018, p. 247). Desde la Sociedad de Puericultura de Buenos Aires se estableció que la asistencia al niño enfermo era sólo una parte de la protección a la infancia y de menor importancia que la prevención del niño sano. Según el médico puericultor Pascual Cervini (1941), comprendía una sucesión de cuidados desde la salud de los padres hasta la adolescencia de los hijos:

La Puericultura es una disciplina que enseña los cuidados necesarios para obtener niños normales, conseguir que ellos realicen un crecimiento y desarrollo también normales. Encierra una sucesión de cuidados de los que buen número se llevan a cabo a través de los futuros padres y muchos más directamente sobre el hijo, desde su nacimiento hasta su adolescencia. Su objeto es un fin noble y sus problemas están íntimamente ligados a la salud y bienestar de la familia, a la grandeza de la sociedad y capacidad y existencia del Estado (p. 59).

Una de sus principales líneas de acción fue la divulgación de reglas y preceptos para una crianza considerada científica, es decir, alejada de las costumbres y saberes populares (Briolotti, 2016a, p. 5). La salud también abarcó 
aspectos relativos a la subjetividad, lo que era bien visto en una madre y su niño, y sirvió para instaurar rituales y prácticas consideradas normales o aceptables.

El desarrollo de las nociones y prácticas que fueron identificadas dentro de la rama de la puericultura fueron indisociables del surgimiento de los dispensarios de lactantes, lugares donde se pudieron llevar a cabo objetivos ligados a la prevención de la mortalidad infantil; en Buenos Aires nacieron en el marco de la "cuestión social". En 1904, el doctor Enrique Foster fundó la Gota de Leche, donde se daban consejos a las madres y se distribuía leche esterilizada (Gaing, 1938, p. 12). En 1898 ya funcionaban en Francia los primeros dispensarios, cuyas actividades llenaban diversas funciones: "una inmediata o asistencial de atraer, diagnosticar y asistir a la población de la zona de influencia y otra más lenta y constructiva de profilaxis y educación. Ambos dirigidos a mantener la salud" (Zwanck, 1943, p. 2). También el médico Juan Murín Navarro (1943) estableció al respecto:

La importancia que tiene el Dispensario en la lucha contra la mortalidad infantil en las zonas de influencia en su calidad de organismo destinado a generalizar el examen y la vigilancia médica del niño y a corregir los defectos de su alimentación, pudo apreciarse desde que Dufour fundó en 1894 la primera institución que se denominó Consulta Gota de Leche. A partir de ese momento según Nobecourt, Ficatelier en Bar le Duc, Alexandre en Pais de Calais y Marois en Yonne pudieron demostrar que en las poblaciones donde se instalaba una de esas sencillas consultas para lactantes la mortalidad descendía de forma inmediata y sorprendente (pp. 48-49).

En 1909, según el doctor Ernesto Gaing, ${ }^{1}$ se crearon tres dispensarios más, uno de ellos dirigido por él, otro por el doctor Klappenbach y otro más por el doctor Pedro Oliverio. Funcionaron como consultorios externos que tenían una dualidad: por un lado, educar a las madres en preceptos de la puericultura $y$, por otro, proveer alimento contra lo que fue llamado en aquella época el peligro alimenticio, es decir, las patologías provocadas por una mala nutrición. También tuvieron objetivos relacionados a intervenir en las formas de vida de

${ }^{1}$ Graduado en 1904, se dedicó a la pediatría y a la puericultura. Creó un alimento hipercalórico para el tratamiento dietético de los lactantes, mezcla conocida con el nombre de leche hipergrasosa de Gaing. Miembro inicial del personal de la Dirección de Protección a la Primera Infancia y jefe del Instituto de Puericultura $\mathrm{N}^{\circ} 2$ (Billorou, 2006, p. 40). 
los sectores más vulnerables y en su moralización (Briolotti, 2016a, p. 1081). Los médicos que trabajaban en los mismos, además de atender a los niños y sus madres, elaboraban diagnósticos y estadísticas sobre temas puntuales.

Por otro lado, la municipalidad contó con lactarios donde mujeres daban el pecho gratuitamente a los niños que lo necesitaban, atento a que sus madres estaban enfermas o bien no poseían recursos suficientes para alimentarlos (Miranda, 2019, p. 160). Además, existieron en Buenos Aires otros organismos que se encargaron de la protección de la primera infancia como la Sociedad de Beneficencia, la cual sostuvo institutos de maternidad modelos, casa de niños expósitos, colonias para niños de diferentes edades para la enseñanza común y de oficios, ayudas a la crianza, colocación familiar del recién nacido. Esto quería decir que los niños eran llevados por una visitadora social a otra familia hasta que tuviera al menos dos años de edad. También prestó asistencia hospitalaria.

Lo primordial en la protección a la primera infancia era proporcionar lo que contribuyera a vigorizar la resistencia del organismo. La principal causa que lo menoscababa era la alimentación inadecuada. Según Gaing (1938):

En la infancia el alimento tiene un triple valor: vigoriza la resistencia, mantiene el organismo y provee a su desarrollo. Para proteger a la infancia hay que mejorar su alimento [...] Para 1909 nuestra elevada mortalidad se producía entonces casi toda a expensas del $25 \%$ del total de lactantes, es decir, de los no criados a pecho. Deducción forzosa: entre nosotros la alimentación artificial se hacía de un modo pésimo y había que buscar el mejor medio de corregirla. Con el reparto de leche esterilizada no se subsanaba mucho. Fue entonces que propusimos la creación de la cocina de leche para tratar de eliminar en lo posible el grave peligro (pp. 12-14).

No todos los dispensarios tenían una cocina de leche; en el caso que la tuviera, se contrataba a personal especializado para su preparación. En 1908, Gota de Leche se transformó en la Dirección Municipal de Protección a la Primera Infancia, dirigida por el doctor S. Oliva, y, luego, tras su muerte, por el doctor Mario Bortagaray. La misma funcionó dentro de la Asistencia Pública de Buenos Aires, que había incrementado, desde principios de siglo, la infraestructura asistencial tanto en la capacidad de internación como en los servicios ambulatorios. La municipalidad de Buenos Aires fue una de las primeras jurisdicciones en incluir en su agenda la problemática de las altas tasas de mortalidad infantil. En la última década del siglo XIx, la Asistencia 
Pública creó el Patronato de la Infancia, institución filantrópica que sostuvo un consultorio médico gratuito para la primera infancia, una sala cuna para los menores de seis años y un internado para niños abandonados. No obstante la acción del Patronato, las autoridades vieron la necesidad de delinear un plan de asistencia a la niñez. Así surgió la Dirección Municipal, la cual tuvo una triple función: médica, educativa y social, a través de sus dispensarios de lactantes e institutos de puericultura (Biernat y Ramacciotti, 2008, p. 334).

Uno de los objetivos de la Dirección fue disminuir la mortalidad infantil. En su órbita llegaron a "funcionar cinco Institutos de Puericultura y veinte dispensarios donde se atienden consultas de lactantes y se distribuyen acciones para los mismos y cuya concurrencia llega a la mitad de los 40000 niños que anualmente nacen en la ciudad" (Schweizer, 1939). Esto era un indicador, según Briolotti (2016a, p. 1082) del éxito de médicos y visitadores de higiene en su tarea de acercar a la población a los dispensarios y familiarizarla con el discurso y prácticas médicas. En 1908, fueron 232 los niños atendidos en los dispensarios, y para 1909 el número aumentó a 860 niños. Hacia 1920 se atendieron a 11415 niños y en 1930 fueron 22 795, casi 100 veces más del total alcanzado el primer año. Para 1940 fueron 21855 niños asistidos, es decir, $24.49 \%$ de los infantes nacidos (Billorou, 2006, p. 88).

En el ámbito municipal también se desarrollaron colonias de vacaciones, comedores infantiles, asistencia odontológica, inspección de nodrizas, colocación familiar del recién nacido de madre tuberculosa y escuela de jardineros. Las principales asistentes de los dispensarios eran mujeres de pocos recursos económicos y, en algunos casos, solteras, para quienes fueron destinadas algunas medidas de los servicios de asistencia social. Un grupo especial fue la madre pobre soltera que fue considerada por los médicos responsable de la disminución de la natalidad y que, al mismo tiempo, necesitaba gran protección y cuidados. A través de las consultas que se ofrecían a las madres en los dispensarios se les proveía de conocimientos que, según los médicos, podían repercutir favorablemente en la salud y en las causas de morbimortalidad. Por ejemplo, que estuvieran atentas a la talla y al peso progresivos de los niños y a los exámenes médicos periódicos. Esto era parte de la instauración de una cultura sanitaria en la que el puericultor era un gran exponente de la medicina preventiva. La labor de la Dirección fue vinculada a la disminución de la mortalidad infantil en menores de cinco años en la ciudad de Buenos Aires. Si para 1900 representó $44 \%$ del total de las defunciones generales, para 1930 disminuyó a 18.2\% (Billorou, 2006, p. 92). 
En las décadas de 1920 y 1930 se impulsó la fundación de institutos de puericultura, los cuales ampliaban las funciones de los dispensarios con la infraestructura necesaria para la internación de la madre y el niño (en recintos separados para mejorar la fiscalización e higiene), ante casos en los que no era posible el tratamiento ambulatorio. Esto permitiría "fortalecer el vínculo de la madre con el hijo, someter a la madre a la disciplina y que esta aprendiera los fundamentos de la Puericultura" (Gaing, 1938, p. 14). El primer instituto de puericultura fue el Número 1 de Güemes, dirigido por Ernesto Gaing; en este se realizaron actividades principales propias de un dispensario de lactantes, como vigilancia y reglamentación dietética, provisión a los lactantes de alimentos adecuados (en raciones preparadas y dosificadas) e inspección a domicilio de visitadoras. Asimismo, se efectuaron prácticas complementarias como la pesquisa de raquitismo, sífilis y tuberculosis, vacunaciones antivariólicas y antidiftéricas, vinculación con maternidades, escuelas de madres y se puso en discusión la posibilidad de dar asistencia médica a domicilio (Gaing, 1938, p. 22). Como ha establecido Nari (2005), la internación de la madre sólo duraba el periodo necesario para el tratamiento de la enfermedad o contingencia que la había provocado y la más común era la falta de secreción láctea. Esta modalidad de atención ampliada fue parte de lo que Ernesto Gaing llamó "protección argentina" a la primera infancia.

Los servicios de puericultura también se establecieron en servicios sociales de maternidades (por ejemplo en el hospital Durand, Beneficencia, Ottamendi, entre otros) y a través de visitadoras quienes, entre 1920 y 1930, se incorporaron a los institutos de puericultura (Briolotti, 2018, p. 182). Ellas se presentaban en las casas de las recién paridas y actuaban como agentes de salud para cuestiones básicas de higiene, estimulando la concurrencia de los sectores más pobres.

La mayor injerencia del Estado respecto de la protección materno-infantil hacia la década de 1920 llevó a diversas acciones para procurar una centralización en el nivel nacional. En 1923 se creó la Sección de Asistencia y Protección a la Maternidad y la Infancia, bajo la dirección de Gregorio Aráoz Alfaro, en el seno del Departamento Nacional de Higiene.

En diciembre de 1936 se sancionó la Ley número 12.341, la cual preveía la creación de la Dirección de Maternidad e Infancia, en cuya esfera se situaron los dispensarios de lactantes y demás instituciones de ese tipo (Briolotti, 2016a, p. 1081). Según Billorou (2006), esta Dirección encontró fuertes resistencias en los médicos municipales, muy poco dispuestos al abandono de su 
autonomía de gestión y decisión. Dentro de los objetivos de esta Dirección estaba el de "perfeccionar" generaciones futuras, combatir la morbimortalidad infantil en todas sus causas y amparar a la mujer en su condición de madre o futura madre (Cervini, 1942, p. 60). Se propuso la asistencia preconcepcional, del embarazo, el parto, la vigilancia del niño a través de fichas sanitarias, lactancia materna, alimentación racional y protección social para niños necesitados (Biernat y Ramacciotti, 2008, p. 335). Estas finalidades estuvieron en consonancia con el fortalecimiento de la eugenesia en su vertiente positiva, la cual se proponía estimular la reproducción de los individuos pensados superiores promoviendo un pueblo sano y vigoroso para defender la patria y dar a luz hijos sanos y fuertes. Desde este punto de vista, las maternidades tenían una finalidad eugenésica y un papel en la lucha contra la morbimortalidad y la mortalidad neonatal, así como en la futura salud del niño: "la protección del lactante no comienza en el nacimiento. Ella es inseparable de la salud de los padres" (Guiraldes, 1944, p. 19). Además, esta prevención debía llevarse a cabo antes del nacimiento a través de la puericultura prenatal, con la atención médica y social a las embarazadas (especialmente las solteras, para quienes se pensaron distintas formas de asistencia social).

En la época estudiada existieron experiencias en España y Brasil que tuvieron orientaciones sobre la puericultura afines a la construida en Argentina en relación con el énfasis en la prevención. En la España de entreguerras se consideró que se debía cuidar al niño antes de su nacimiento a través de la puericultura preconcepcional o la eugenesia, con especial preocupación por asegurar la lactancia materna y la adecuada nutrición (Bernabeu Mestre, Trescastro López y Galiana Sánchez, 2011, p. 50). En Brasil, la creación del Departamento Nacional de Crianza en 1940 fue una expresión de la perspectiva social que asumió el discurso de los puericultores, para lo que se acercaron a la retórica eugénica y a la tesis neolamarckiana sobre las propuestas de intervención en el medioambiente, partiendo de que el adecuado cuidado de los niños podía "regenerar" la nación (Lópes y Maio, 2018).

\section{SOCIEDAD DE PUERICULTURA DE BUENOS AIRES. PRESENTACIÓN DE LOS ANALES}

La Sociedad de Puericultura de Buenos Aires fue fundada el 14 de septiembre de 1934 con personal de la Dirección Municipal de Protección a la Primera 
Infancia de Buenos Aires y se ubicó en la calle Victoria número 1089. Uno de sus principales impulsores fue el doctor Alfredo Buzzo, quien la dirigió entre 1934 y 1937. Fue jefe del Dispensario de Lactantes Número 5 y profesor de Toxicología de la Facultad de Medicina de la Universidad de Buenos Aires.

Los motivos del surgimiento de la Sociedad estuvieron relacionados con la necesidad de generar un espacio científico destinado a los saberes sobre la Puericultura y, a la vez, fortalecer su papel institucional a través de la labor llevada a cabo en dispensarios e institutos de puericultura existentes. Tuvo características particulares que la diferenciaron de otras sociedades científicas de la época, ya que reivindicó su autonomía, pero sin enfatizar en que fueran un conjunto de hombres dotados de conocimientos especiales con condiciones morales de una elite, sino como condición de la eficacia de un trabajo técnico destinado al cumplimiento de una función social prioritaria (Billorou, 2006, p. 32).

Dentro de los objetivos establecidos por la Sociedad en su acta de fundación figuraron, en primer lugar, auspiciar el mejoramiento de la protección a la primera infancia; en segundo, desarrollar su función social y, finalmente, promover reuniones de carácter científico para ahondar en lo relativo a la puericultura, lo cual implicaba el aumento de sus integrantes. Para lograrlo, se establecieron diversas categorías de socios, entre ellos, los socios adherentes, que eran los médicos que trabajaban en otros servicios de puericultura que no dependían de la Dirección Municipal. Igualmente, se amplió la condición de socios titulares a dirigentes de instituciones en las que se profundizaran los problemas de la puericultura y especialidades afines (Billorou, 2006, p. 46). Con la categoría de socios correspondientes se posibilitaba la integración de médicos nacionales y extranjeros a pesar de no vivir en la Capital Federal. También había socios honorarios, quienes fueron referentes con una actuación prestigiosa en la puericultura; entre ellos, el médico pediatra Gregorio Aráoz Alfaro y Juan Pedro Garrahan, profesor titular en la Cátedra de Clínica Pediátrica del Hospital de Clínicas (1942-1962) y organizador y jefe del Departamento de Puericultura del Instituto de Maternidad de la Sociedad de Beneficencia, quien sostuvo un enfoque que propuso adaptar los consejos de los médicos a la particularidad psicofísica de cada niño y sus variables emocionales en su libro La salud del hijo, publicado en 1955 (Briolotti, 2016b, p. 51).

Otro de los miembros honorarios fue Alberto Zwanck, profesor de Higiene y Medicina Social de la Facultad de Ciencias Médicas de la Universidad de Buenos Aires, médico inspector del Consejo Nacional de Educación 
y del Departamento Nacional de Higiene. De igual forma integró la nómina Fernando Schweizer, profesor titular de Clínica Pediátrica y Puericultura de la Facultad de Ciencias Médicas de La Plata.

Asimismo, Ernesto Caece (Nápoles), creador de la Nipiología como rama dedicada a solucionar la tutela higiénica de los niños de primera edad, entendida como la ciencia integral del lactante, cuya repercusión en Argentina llevó a la fundación de la Sociedad de Nipiología e Higiene en 1922, presidida por el médico Mamerto Acuña, quien también fue miembro honorario (Miranda, 2019, p. 162). Además, Benjamin Weill-Hallé (pediatra y puericultor francés, director de la Escuela de Puericultura de París, discípulo de Adolphe Pinard, encargado del Curso de Puericultura en la Facultad de Medicina de París y director de la Revue Francaise de Puericulture), entre otros médicos.

Desde la Sociedad de Puericultura de Buenos Aires se formó una biblioteca especializada con libros nacionales y extranjeros, y en 1935 se fundó la publicación Anales de la Sociedad de Puericultura de Buenos Aires, la cual permitió la generación sostenida de producción científica especializada, difusión de eventos y noticias académicas. Fueron publicados de manera trimestral para difundir conocimiento sobre la puericultura. Sus secretarios de redacción fueron Adelcira Agostini de Muñoz (perteneció a la primera generación de médicas argentinas y trabajó en el hospital Durand, donde funcionaba el Instituto de Puericultura Número 2), Fernando Ugarte y Delio Aguilar Guiraldes. Los miembros de la redacción incluyeron al cuerpo médico de la protección de la primera infancia municipal. En 1940 cambió el equipo, el cual estuvo constituido por el doctor Isidro Puig, Alfredo Vidal Freyre, Santos L. Crespi, Juan V. Tiscornia y Mario Waismann. Todos los miembros honorarios de la Sociedad fueron consultores externos de Anales, cuyos nombres figuraron en la contratapa de la publicación.

Anales estuvo dirigida a médicos, con un lenguaje técnico, a diferencia de otras publicaciones de la misma época como Hijo Mío!..., las cuales eran dirigidas a madres y contenían consejos sobre crianza. En 1943, Anales dejó de existir y en su lugar se publicó la Revista de la Sociedad de Puericultura de Buenos Aires (1944-1951), incluso dirigida por el médico Samuel González Aguirre, cuando perdió apoyo financiero e institucional.

A partir de abril de 1941 se incluyó en Anales una sección con sesiones ordinarias y extraordinarias de la Sociedad Argentina de Pediatría. Además, contaba con la de "Libros y revistas", con reseñas de libros nacionales y extranjeros, "Crónica e informaciones" y un índice en la página final. Su extensión 
fue de aproximadamente 100 páginas por tomo. En "Crónica e informaciones" se publicitaron diversos eventos académicos como el $5^{\circ}$ Congreso de Pediatría, en 1940, en Boston, Estados Unidos, en el cual Gregorio Aráoz Alfaro, Mamerto Acuña, Juan P. Garrahan, Fernando Schweizer, entre otros, integraron el comité organizador. Por otra parte, sobresalieron los temas oficiales del Primer Congreso Nacional de Puericultura, llevado a cabo del 7 al 11 de octubre del mismo año en Buenos Aires, y fueron los siguientes: crecimiento y desarrollo físico y psíquico en los niños, raquitismo, legislación y asistencia. Los temas recomendados: profilaxis de la tuberculosis y de la sífilis (Primer Congreso Nacional de Puericultura, 1940, p. 149). También se dio cuenta de la creación, en 1937, en el Hospital de Niños Expósitos de una cátedra libre de puericultura a cargo del médico Pedro Elizalde "para combatir la alta mortalidad y la morbilidad infantil de los niños de primera infancia, especializando a los egresados de la Facultad de Medicina de la Universidad de Buenos Aires para que puedan cuidar con éxito a los lactantes" (Schweizer, 1939, p. 20). Desde esta cátedra se dictó en 1940 un curso sobre tuberculosis en la primera infancia con clases teórico-prácticas (Cátedra de Puericultura. Curso para posgraduados, 1939, p. 308). Aparecieron avances institucionales de otros países, como la creación del Instituto Nacional de Puericultura brasileño, bajo la dirección del profesor Martagao Gesteira (Creación del Instituto de Puericultura brasilero, 1938, p. 73). Fueron divulgadas las Bodas de Plata de la Sociedad Uruguaya de Pediatría (1943, p. 117), sus sesiones científicas y banquetes a los que asistieron, como representantes de la Sociedad, Gregorio Aráoz Alfaro, Mamerto Acuña, Pascual Cervini y Jaime Damianovich, entre otros.

El director de Anales fue Carlos Montagna entre 1937 y 1940, discípulo de Pedro Escudero (1877-1963), quien, junto con sus colaboradores, fue pionero en el desarrollo de investigaciones académicas sobre la nutrición en Argentina y alertó a las autoridades políticas y miembros de la sociedad civil sobre las consecuencias negativas de una mala alimentación, quienes impulsaron espacios de formación profesional. En particular, fue relevante la creación del Instituto Municipal de Nutrición en 1928 (Buschini, 2013, p. 135). La calidad de la leche de vaca brindada a los niños en los dispensarios puede ser encuadrada dentro de esta preocupación, dado que desde este Instituto se realizaron investigaciones y tareas asistenciales al respecto. Asimismo, Escudero creó una mezcla láctea para bebés que llevó su nombre. Montagna trabajó bajo su dirección en la Escuela de Dietistas y en el Instituto Municipal de Nutrición. Fue jefe del Dispensario de Lactantes número 7 y luego del 
Servicio de Niños del Instituto Municipal de Nutrición. Su libro Puericultura. Higiene y alimentación del niño (1947) constituyó uno de los referentes en la especialidad (Billorou, 2006). La formación de Montagna tuvo ciertas implicaciones en la elección de los temas de los artículos de Anales, ya que surgió de forma notoria un interés por cuestiones alimentarias en los lactantes durante su dirección y de trabajos al respecto elaborados en el Instituto Municipal de Nutrición. En 1941 pasó a dirigir la publicación Anales el médico Jaime Damianovich y, entre 1942 y 1943, Alfredo Vidal Freyre, quienes trabajaron en el ámbito de los dispensarios municipales.

Es posible que Anales haya circulado por los espacios de la Dirección Municipal de Protección a la Primera Infancia, donde se situó institucionalmente la Sociedad y la Facultad de Medicina de la Universidad de Buenos Aires, donde eran profesores algunos miembros del Comité Honorario de la Sociedad. Asimismo, en el ámbito de casas de productos dietéticos, atento a las publicidades que sostuvieran la publicación, además de los aportes de los socios. A esta publicación se podía acceder a través de una suscripción anual de cinco pesos.

Dentro de las publicidades que aparecían en Anales se encontró Galactosi, producto presentado como capaz de aumentar la secreción de la leche materna con propiedades tonificantes del glicerafostato de sodio; Cremil, leche ácida hipergrasosa (para niños sanos desde el nacimiento); leche Degerma, la cual cumplía todas las exigencias de una leche sana e higiénica para niños y adultos, sanos o enfermos. Se aclaró que provenía de vacas sanas bajo continuo control veterinario, ordeñada limpia e higiénicamente por personal instruido, homogeneizada para su mayor digestibilidad y determinada en el mismo envase de venta para protegerla contra eventuales infecciones. Además, esta leche se repartía de forma diaria a domicilio en envases de un litro, previo pedido telefónico. Durante este periodo hubo debates en torno a la regulación estatal para la comercialización de la leche de consumo en Buenos Aires, alimento considerado como un vehículo de enfermedades como la fiebre tifoidea, cólera, tuberculosis y podía llevar a los niños pequeños a peligrosas diarreas o la muerte. En particular, hubo disidencia sobre la pasteurización obligatoria $y$, en el caso de algunos expertos como Escudero, recomendaron que en los hogares hirvieran la leche como método eficaz para eliminar agentes patógenos y saprófitos sin alterar el valor nutritivo (Buschini, 2018, p. 40). También apareció Soybee, producto dietético para los casos de alergia a la proteína de la leche de vaca, huevos y cereales, y Sanasoy, harina de soya para aplicación médica en casos de diabetes, desnutrición, pielitis, etc. Nestlé fue otro de los 
auspiciantes de Anales con sus productos Nestógeno, leche semidescremada en polvo y leche condensada (azucarada condensada al vacío). En 1939 la marca donó una serie de libros provenientes de Francia sobre nutrición y niñez a la biblioteca de la Sociedad. Su inclusión toma relevancia porque la invención de la fórmula de leche estuvo a cargo del farmacéutico Henry Nestlé en 1867 y consistía en una mezcla de leche de vaca, harina y azúcar (Gimeno, 2018).

\section{DISCURSOS Y PRÁCTICAS EN TORNO A LA LACTANCIA EN ANALES DE LA SOCIEDAD DE PUERICULTURA DE BUENOS AIRES (1935-1943)}

En los primeros años, Anales se limitó a publicar las comunicaciones discutidas por sus asociados en las sesiones ordinarias de la Sociedad, las cuales, en muchos casos, se convertían en artículos publicados. En estas transcripciones aparecieron las voces de los médicos sobre cómo resolver cuestiones asistenciales, su conveniencia o viabilidad práctica (en casos de tratamientos de patologías o trastornos en lactantes) y se dirimieron posturas teóricas. Se trataron temas como la lactancia natural y aversión al seno materno, llanto del lactante, extracción artificial de leche materna, dentición y alimentación, difteria, tratamiento para el raquitismo, bocio, conjuntivitis, ictericia, entre otros. Los debates generados fueron parte de la problematización de la lactancia, es decir, de su constitución de cierta forma, caracterizada con elementos singulares e implicando formas específicas de intervención.

En los discursos de Anales fue nodal el papel de la lactancia y de la leche como alimento, y las tecnologías de gobierno implementadas en torno a la lactancia estuvieron orientadas a restablecer y conservar la salud integral, en especial la digestiva, de los niños recién nacidos y de pocos meses. Además, los médicos, a través de la educación a las madres sobre cómo dar pecho o leche esterilizada (su adecuada conservación y preparación), ponían en marcha tecnologías que tuvieron como uno de sus principales efectos reorganizar los comportamientos de las madres y asegurar la adecuada calidad de la leche artificial brindada. Los médicos recomendaron la lactancia materna, la cual, según Gimeno, constituye una práctica biosocial y un símbolo de vida frente al flagelo de la desnutrición, la muerte y la degeneración. Además, permitía prolongar el lazo vital de la madre con el hijo como una unidad funcional de base biológica. Para Gimeno (2018): 
La lactancia puede considerarse una institución clave en la reproducción de la sociedad y de la cultura que ha dado lugar a diferencias de clase, a distintos rituales domésticos y sociales y formado parte de la condición histórica y social de las mujeres. El tipo de maternidad que se practique o sea socialmente dominante en cada momento es lo que va a determinar cómo se practique, cuándo y cómo y ambas van a estar a su vez relacionadas con la situación de las mujeres en cada sociedad (p. 86).

La lactancia materna apareció como una obligación femenina de la que sólo se podía exceptuar a la mujer por cuestiones médicas, y en situaciones especiales: los casos de niños con necesidades específicas como los prematuros, con distrofias (es decir, con problemas a nivel muscular para la succión) o mellizos que requerían un complemento alimenticio para sostener su salud y llegar a la talla de crecimiento considerada normal.

Las indicaciones de los médicos a las madres no se limitaron a cómo y cuándo dar el pecho, sino también a cómo nutrir al niño adecuadamente en el caso que esto no fuera posible a través de leches comerciales, de fórmula y complementos vitamínicos. La adecuada alimentación era parte, a la vez, de inculcar contenidos culturales difundiendo normas de higiene pública y privada (Donzelot, 2008). Además, brindar leche artificial puede encuadrarse dentro de lo que fue llamada la alimentación científica, es decir, aquella que se planifica racionalmente según las necesidades de nutrición de cada individuo, de su costo en términos económicos y de su palatabilidad, es decir, que sea apetecible. Esto puede entenderse en consonancia con lo que ocurría a nivel mundial en el periodo de entreguerras, en el que las pautas para la selección y consumo de alimentos estarían dictadas por expertos y tenderían a ser homogeneizadas, en detrimento de criterios tradicionales y culturalmente diversos (Andreatta y Suárez, 2013, p. 157). Desde Anales, como establecieron Adelcira de Muñoz y María Escudero (1937), para alimentar a los bebés antes de la extensión de la leche vacuna:

Históricamente los indios de las praderas y los esquimales del continente americano, utilizaban galleta de yuca y de maíz, mate y aún cacao, el que reducían a polvo y le agregaban azúcar confeccionando el chocolate que preparaban con agua: desconocían el uso de la leche vacuna, sí la de llama, alpaca, vicuña y sus derivados. En el siglo XVIII la actividad referente a higiene infantil se va 
intensificando y procurando la dietética, se inicia la propaganda sanitaria por los médicos y los folletos instructivos para las madres (p. 264).

En 1932, el Comité de Salud de la Liga de las Naciones se pronunció sobre los requerimientos en la dieta según edad, sexo y ocupación; especialmente una sección fue para embarazadas y niños, así como una escala de coeficientes para su adecuación a cada caso. Desde Anales, la nutrición era una cuestión científica de la que el puericultor debía tener conocimiento para llevar a cabo su tarea. Según Gaing (1942, p. 47), era en la primera infancia donde los procesos de nutrición y crecimiento adquirían su grado máximo, época de más rápido crecimiento, y cualquier perturbación de su normal desarrollo repercutía de un modo desconocido en la vida adulta.

En los discursos de Anales se constataron diferencias entre los niños alimentados artificialmente y los alimentados a pecho, ya que, según algunas experiencias, los primeros aumentaban mejor de peso y eran más sanos en general. En la sección de reseñas de "Libros y revistas" se publicó un análisis de un artículo publicado en los Archivos de Pediatría de Uruguay (1940) con motivo del 50 aniversario de la alimentación artificial del lactante por el reconocido médico Finkelstein:

La estadística de Boeck hace más de 60 años demostraba que la mortalidad de los niños criados artificialmente era 7 veces mayor que la de los criados a pecho. Se creyó que la contaminación de la leche de vaca sería la causa, pero el empleo de leche esterilizada no mejoró en gran forma los resultados. Se recomendó entonces la leche cruda, recogida en las mejores condiciones de asepsia, sin mayor éxito. La albumina fue incriminada como causal, luego se demostró que no era así. En forma empírica se han buscado procedimientos mejores de alimentación. Todos ellos pueden reducirse en leche diluida o leche total, importancia del agregado de hidratos de carbono y diferencia entre los diversos hidratos de carbono, importancia del agregado de grasas, leches acidas. Los aportes a la práctica de alimentación artificial han consistido en aprender a conocer las fallas de los antiguos procedimientos de alimentación artificial, aprendiendo evitarlas: hipoalimentación, hiperalimentación y alimentación unilateral (p. 49).

Los médicos que trabajaron en los dispensarios identificaron ventajas económicas en la lactancia materna, ya que no generaba gastos extras en las 
familias. Por ese entonces, las fórmulas de leche en polvo y las pasteurizadas eran costosas. Otra preocupación de los médicos era la preparación de las mezclas de leche para recién nacidos en regiones donde se carecía de leche fresca. Carlos Montagna y Horacio Burgos (1940), desde el Servicio de Niños del Instituto Municipal de Nutrición, establecieron:

Luego de seis años de diaria utilización en millones de niños que concurren al Servicio de Niños y a sus consultorios externos y accediendo a la consulta de médicos de regiones donde no poseen en buenas condiciones leche fresca, hemos preparado las fórmulas para obtener la mezcla láctea. Sólo existe un inconveniente y es su precio, pero sólo la usamos en el primer trimestre de vida en sustitución a la leche materna, cuando esta no se obtiene, el dispendio en realidad es relativo. Tienen una composición porcentual comparable a la leche humana, el valor calórico total por litro es el mismo, los glúcidos, proteicos y lípidos tienen la misma relación por cien calorías en ambas leches (pp. 98-101).

La lactancia tenía innumerables ventajas médicas y biológicas que eran difícilmente asimilables con la leche de vaca. Una de ellas es que se adecuaba a las capacidades digestivas del niño. Los médicos de los dispensarios de lactantes números 7 y 16, Julio Virasoro y Félix Justo Roca (1939), concluyeron, después de observaciones y tratamientos practicados, que la alimentación también tenía un enorme papel en las diarreas, "ya sea por hipo o hiperalimentación, defectos dietéticos o a veces solamente por el destete y el paso a la alimentación mixta o artificial” (p. 279).

Una de las opciones dentro de la leche artificial fue la leche cítrica. Juan Murtagh y Carlos A. Riviere (1943) la recomendaron:

Creemos útil el estudio de alimentos cuya conducta frente a los procesos digestivos gástricos e intestinales, los asemeja a la leche humana, hoy por hoy, desideratum inigualable en la alimentación de los primeros meses de vida. La experiencia de una serie de casos seguidos en el dispensario y en la clientela civil nos ha fortalecido en la convicción de que la leche acidificada con jugo de limón constituye un alimento muy digno de ser tenido en cuenta por su tolerancia, facilidad de preparación y los resultados obtenidos (p. 24).

Se reconocieron en la leche ácida beneficios, al producir una rápida y fácil digestión, contribuir al normal funcionamiento pilórico y favorecer en 
el duodeno la actividad de la secretina. Las ventajas eran su fácil obtención y preparación en el ambiente familiar, simplificar y asegurar con su empleo el aporte vitamínico necesario y por producir una más fina floculación de la caseína. El principal inconveniente era el rechazo por parte de la criatura, por lo que los médicos insistían en que debía ser administrada a niños de pocas semanas, quienes la aceptaban de buen grado, y llegaban a aficionarse tanto a ella que la podían preferir a otras formas de alimentación. En los lactantes mayores, el rechazo era más frecuente. Murtagh y Riviere (1943) señalaron:

Nuestra experiencia se basa en unos 40 casos seguidos en el Dispensario $\mathrm{N}^{\circ} 10$ y unos 60 de nuestra clientela civil. Desde hace 3 años indicamos casi sistemáticamente la leche cítrica en todos los niños alimentados artificialmente desde el mes de edad. Es un alimento económico y, en este sentido, superior a la leche entera en polvo, acidificada. Sin embargo, en ciertos ambientes, en determinadas épocas del año, la adquisición de un limón diario representa un verdadero sacrificio. Esto limita en parte la universalidad de la aplicación. La técnica de preparación consiste en agregar el jugo de limón a las dosis de $5 \%$ en la leche hervida, enfriada a $29^{\circ}$ gota a gota o bruscamente, agitando bien (p. 30).

Estas propuestas nutricionales y sus ventajas, así como las posibilidades de asimilarla a la leche materna, remiten a un dinamismo y coexistencia entre distintas modalidades de alimentación: la lactancia como principal y, como segunda opción, leches comerciales para niños sanos o con necesidades específicas y fórmulas elaboradas por los médicos como la de Escudero, de Gaing o económicas, como el último caso. En sí, configura parte de la invención de técnicas para asegurar la salud de los lactantes, en las cuales se asumió que era parte del papel de la puericultura el estudio de las leches que fueran digeribles.

Además de la alimentación de pecho o artificial, los médicos Mendilaharzu, Gentile y Crouzel (1942) plantearon el papel de las vitaminas en los niños pequeños:

Está bien demostrado que no sólo la ración calórica debe ser cubierta, ya que la carencia de elementos como las vitaminas y aminoácidos detienen el crecimiento y traen trastornos múltiples. La labor en los últimos años de estudios sobre la constitución química de varias vitaminas ha proporcionado novedades importantes en el estudio de la patogenia de ciertas enfermedades. No sólo como substancias complementarias sino por sus efectos terapéuticos (p. 263). 
Mendilaharzu, Gentile y Crouzel (1940) llegaron a distintas conclusiones al respecto, entre ellas, que el lactante de pecho disponía, por ejemplo, de una cantidad de ácido ascórbico más que suficiente para cubrir las necesidades del organismo. "El niño alimentado con leche de vaca no siempre está libre de trastornos de hipovitaminosis $\mathrm{C}$, presentándose estos a consecuencia de diferentes enfermedades" (p. 112).

Muchas leches artificiales que circularon en Buenos Aires fueron fuente de problemas, como establecimos anteriormente, y los médicos en estos dispensarios, a través de las fórmulas Escudero, Gaing y leches cítricas, tuvieron como objetivo reducir riesgos relativos a su conservación y composición. Con el amamantamiento era nulo el peligro de portar microbios responsables de enfermedades infecciosas, como sucedía con el biberón. Al respecto, en Anales apareció un proyecto del médico Guillermo Bayley Bustamente (1941) presentado en las sesiones de la Sociedad, para la conservación de la leche artificial:

Observo la relativa frecuencia con que las diluciones lácteas entregadas a la mañana por la Cocina de leche, se alteran en el domicilio de los niños, durante las horas de la tarde y que son a mi juicio tres las razones: la calidad de la leche provista, la competencia del personal, las condiciones en que se conserva el alimento en el domicilio de los niños. Propone un envase refrigerante a hielo seco que facilita la conservación de las mezclas lácteas y es económico y de fácil realización (pp. 46-47).

La iniciativa fue celebrada en las sesiones por otros miembros de la Sociedad como el doctor Jaime Damianovich, quien estableció que era necesario disponer de fondos para someter a ensayo el dispositivo propuesto. El presidente de la Sociedad, Pascual Cervini, cuestionó la importancia del papel bacteriológico en la leche hervida, como fuente de trastornos en el lactante. Su impresión personal era que desempeñaba un papel secundario en el determinismo de esos procesos. Es decir, que era más importante que la leche estuviera esterilizada, por lo que propuso una comisión especial, integrada por los médicos José Vidal Freyre, Tomás Sletch y Guillermo Bayley Bustamante, para que se pronunciaran sobre el costo económico del dispositivo refrigerante. Esta propuesta formó parte de los trabajos destinados a impulsar mejoras e innovaciones en la asistencia cotidiana del niño y la madre dentro de la estructura sanitaria municipal de Buenos Aires, desde los espacios científicos de Anales. 
La mayor parte de los artículos publicados en Anales fueron aquellos que eran resultado del trabajo de los médicos en los dispensarios de lactantes. En los mismos apareció una preocupación por la instauración de tecnologías de prevención de la salud del niño lactante y su madre, las cuales estuvieron dirigidas a evitar la mortalidad infantil, la desnutrición, malnutrición y enfermedades degenerativas o venéreas como la sífilis y tuberculosis. En estas intervenciones subyació un interés sostenido por asegurar la vigorosidad y fortaleza del cuerpo social, el cual, en muchos casos, contó con un discurso autoritario. Los médicos Ernesto Gaing (1938) y Juan Murín Navarro (1943) utilizaron léxico castrense para referirse a la protección a la primera infancia. El segundo, que fue el médico fundador de la Caja Municipal de Asistencia Social de la Maternidad e Infancia de Godoy Cruz, Mendoza, estableció:

El Dispensario constituye el arma específica de la medicina social de la infancia como problema de masas, $y$ dentro de la medicina de masas, como dentro de la lucha contra cualquiera de las enfermedades sociales, la solución asistencial no radica en llevar el maximum de perfeccionamiento a algunos sitios y a grupos reducidos de individuos, sino, de encontrar el medio de asegurar el minimum de corrección en todas partes y al mayor número posible de habitantes. Podemos afirmar que el Dispensario es al problema de la Sanidad, lo mismo que la escuela es al problema de la enseñanza (p. 12).

Gaing publicó en 1938 un artículo titulado "Nuestra protección municipal a la primera infancia”, en el cual historizó la creación de los dispensarios en la ciudad de Buenos Aires y describió su trabajo en los mismos. Según él, la protección a la primera infancia debía ser "totalitaria", retomando este concepto de un general militar:

Uno de los militares que más se distinguieron en la guerra mundial por su talento y planes estratégicos, el general Ludendorff, escribió un notable libro en el que expone las medidas que habría que tomar en la lucha contra el enemigo y el modo de disponer de todas las fuentes y agitar todos los recursos ya militares, económicos, políticos o administrativos, para obtener el triunfo si desgraciadamente se desencadenaría una nueva contienda. Denominó a su obra "Der totale Krieg" en castellano "La guerra totalitaria". Pretendemos aplicar el mismo criterio a la Protección a la primera infancia. Ella también debe ser totalitaria. La mortalidad infantil está constituida por una serie de 
factores que pueden ser considerados como "enemigo" contra el cual debemos, para batirlo, apelar a la totalidad de recursos a nuestra disposición (p. 11)

Para Gaing, el establecimiento de una protección totalitaria a la primera infancia se relacionó con que la función del dispensario era mucho más que proveer de leche artificial o dar consejos a las madres; era un dispositivo de prevención y asistencia que aspiraba a ser total de la salud del niño recién nacido. También de la mujer y de aquellos condicionantes económicos y sociales que podían tener incidencia en la crianza, como fue el caso de las mujeres trabajadoras. Esto, en la práctica, implicaba, por ejemplo, ampliar las funciones de los dispensarios. En el marco de esta idea es que se desarrollaron los institutos de puericultura que Gaing llamó el "tipo argentino" de protección a la primera infancia, compuesto de un dispensario con su cocina de leche anexa, visitadora, internado para madre e hijo, amas y laboratorio de análisis, atendido por personal especializado.

Por otra parte, teniendo en cuenta que las principales destinatarias de los dispensarios eran madres que trabajaban y con pocos recursos económicos, aparecieron propuestas en Anales para garantizar su asistencia y asegurar la lactancia materna. Los médicos Carreño y Cosin (1940) plantearon que los dispensarios existentes funcionaran como hospedaje para los hijos de madres que trabajaban en sus domicilios. "Siendo que las salas maternales establecidas por la ley no han dado el resultado previsto hay que modificarlas agregando una creche o sala cuna para el hospedaje infantil mientras la mujer trabaja. En los casos de mujeres que trabajan en sus hogares los Institutos de Puericultura podrían organizar la nueva sección encargándose del cuidado de estos niños" (p. 116).

La atención de los niños en los dispensarios durante el horario laboral redundaría, según estos médicos, en beneficios para ellos -quienes de otra forma quedaban al cuidado de familiares en lugares muchas veces con hacinamiento-, como el moral, por la tranquilidad de la cercanía con el niño y el cuidado que le iban a brindar en el dispensario. En este periodo habían surgido propuestas legislativas para garantizar condiciones materiales y morales de las mujeres que trabajaban y eran madres. La Ley 11.317 contempló el caso de la obrera madre con su hijo en la circunstancia de trabajar en establecimientos o fábricas y le concedía el derecho de disponer de un intervalo de quince minutos cada tres horas para la lactancia de su hijo, según el artículo 15, primera parte. Disposición modificada por una ley posterior, la 11.932, la cual expresó el 
principio sancionado en la Convención de Washington y ratificado por el Congreso Nacional por la Ley 11.726, la cual indicaba que toda madre de lactante podía disponer de dos descansos de media hora para amamantar a su hijo en el transcurso de su jornada de trabajo, salvo el caso que un certificado médico estableciera un intervalo menor (Carreño y Cosin, 1940, p. 114). La reglamentación nacional y la de la provincia de Buenos Aires establecieron que debía habilitarse una sala maternal adecuada durante el tiempo de ocupación de las madres en todo establecimiento donde se ocuparan 50 mujeres o más, mayores de 18 años. Para los establecimientos donde trabajaran menos de 50 mujeres no estaba regulada legalmente la existencia de una sala como deber del empleador. Carreño y Cosin propusieron que se crearan salas de lactancia bajo la coordinación de la Dirección Municipal de Protección a la Primera Infancia, ya que, hasta entonces, se encontraban bajo la órbita del Departamento Nacional de Trabajo. Dependencia que no tenía en cuenta, según estos médicos, aspectos sanitarios y de atención especializada, como sí lo tenía la Dirección.

Por otro lado, desde los discursos de Anales se realizaron propuestas para mejorar la atención en los dispensarios, lo que implicó medidas específicas como la necesidad de construir boxes de aislamiento para tosedores. Bogani (1941) señaló que: "Los lactantes que son llevados a la consulta para controlar su peso y su alimentación, con un desarrollo óptimo hasta ese momento, adquieren en la sala de espera una afección que acarreará en ellos trastornos, muchas veces graves, por una evolución infortunada. Es prioridad del médico puericultor evitarles esos contactos infectantes que comprometen su salud y ponen en grave riesgo su vida" (p. 26).

Hubo gran cantidad de artículos que eran fruto de extensos trabajos empíricos de los médicos dentro de los dispensarios de lactantes. Por ejemplo, Jaime Damianovich (1938) estudió los efectos de la leche ácida hipergrasosa en 333 casos de niños en su primer trimestre de edad. Los médicos Tiscornia, Waismann y Crespi (1939) investigaron sobre la sífilis: "Cuatro años de trabajo en la pesquisa desarrollada en el Dispensario $\mathrm{N}^{\circ} 4$ y ahora en el $\mathrm{N}^{\circ}$ 19 bajo la dirección del doctor Pascual Cervini. Sobre 2716 niños vistos, desde el año 1936 hasta la fecha, hemos encontrado 39 casos de sífilis congénita, o sea un 1.43 por ciento en total" (p. 271). Los médicos Julio Virasoro y Félix Justo Roca (1939) hicieron un trabajo acerca de la diarrea, el cual era fruto de la investigación de cinco años en los dispensarios números 7 y 16 . Estudiaron un total de 6366 casos de diarreas de lactantes desde un punto de vista clínico y bacteriológico (p. 278). 


\section{REFLEXIÓN FINAL}

Para finalizar, podemos establecer que la puericultura ocupó un lugar clave en relación con las iniciativas para la prevención de la mortalidad infantil, la asistencia del niño y la madre en Buenos Aires a través de los dispensarios de lactantes e institutos de puericultura creados. Anales, como órgano de difusión de la Sociedad de Puericultura de Buenos Aires, fue un espacio de producción científica y laboratorio en la generación de propuestas para mejorar la atención materno-infantil. En particular, de tecnologías de gobierno respecto de la lactancia orientada a restablecer y conservar la salud, en especial la digestiva, de los niños recién nacidos y de pocos meses, así como guiar el comportamiento de las madres respecto a la forma de alimentar y conservar los alimentos. La importancia del estudio de los discursos de la publicación Anales radica en que hizo un aporte específico en la discusión por la adecuada alimentación de los niños y por la difusión de la prevención de la salud, a partir de extensos trabajos empíricos en su trabajo diario en los dispensarios de lactantes e institutos de puericultura de la Dirección Municipal de Protección a la Primera Infancia. La nutrición del lactante fue considerada una cuestión de la que el puericultor debía tener conocimiento para llevar a cabo su tarea.

Una coincidencia entre los articulistas fue que se buscaron evidencias de las ventajas de la lactancia respecto a la leche artificial, tanto económicos (no generaba gastos extras en la familia), digestivos (los niños de pecho tenían menos diarreas o problemas gástricos) y mejor desarrollo en general. Esto es paradójico porque uno de los auspiciantes de Anales fue Nestlé y otras empresas de productos lácteos y vitamínicos destinados a bebés que no tomaban el pecho que fueran sanos o con necesidades especiales (como alergias a la proteína de la leche de vaca, diabetes, desnutrición, etc.). Lo anterior tiene relación con la necesidad de una nutrición de tipo científica en la que el Estado debía intervenir para asegurar su calidad. En Buenos Aires, específicamente, hubo debates en espacios médicos sobre la regulación estatal de la comercialización de leche de consumo a través de la pasteurización, para evitar patologías en los niños. Las leches que circularon en el mercado eran costosas y los médicos, desde los dispensarios, insistieron en lograr fórmulas de leches económicas que los niños pudieran digerir o asimilar, así como en reemplazos vitamínicos. Esto puede encuadrarse dentro de un tipo de higiene positiva ocupada de asegurar buenas condiciones físicas de la población y, en particular, de la primera infancia. 
Una de las cuestiones que guiaron la labor de la Sociedad y su publicación Anales, como su principal órgano de difusión, fue procurar transformar el saber científico en políticas concretas para la protección del niño desde su nacimiento (Billorou, 2006, p. 29). En los dispensarios de lactantes se desplegaron diversas tecnologías de prevención y atención sanitaria para los niños y sus madres. La puericultura fue parte de la instauración, en términos más generales, de una cultura sanitaria. Se centró, en gran medida, en la necesidad de instruir o disciplinar a la madre en cuestiones prácticas de cuidado, higiene y alimentación, construyendo de esta forma subjetividad y un modelo de maternidad deseable. En particular, surgieron las recomendaciones por sostener la lactancia materna, la cual también fue considerada una obligación de la que sólo podían exceptuarse en los casos de indicación médica o específicos (casos de mellizos o prematuros, por ejemplo).

Desde Anales se dieron a conocer propuestas relativas al mejoramiento en la atención en los dispensarios. Una de ellas fue la Carreño y Cosin, de ofrecer asistencia en salas cuna para madres trabajadoras para asegurar la lactancia. También la de construir boxes para tosedores o de un envase refrigerante con hielo seco para garantizar la conservación de la leche brindada en los dispensarios. Estas medidas fueron parte del acento en la prevención y acorde con el perfil local de la puericultura respecto de que los cuidados estaban destinados a todos los niños, pero, sobre todo, a los sanos. Con su fortaleza se aseguraba la del cuerpo social. En la asistencia que se brindaba a través de los dispensarios de lactantes e institutos de puericultura surgió un sesgo autoritario a través de categorías que utilizaron los médicos como protección "totalitaria" esbozada por Ernesto Gaing. Esta expresión remitió a la ampliación progresiva en los alcances y funciones sanitarias y sociales de los institutos de puericultura para cubrir de manera total la asistencia de la madre y el lactante, convirtiéndolos en un dispositivo más complejo. Esto fue parte del contexto social y cultural en el que habían adquirido importancia las nociones eugenésicas, mismas que servían de fundamento científico de la necesidad de la vigorosidad y fortaleza del cuerpo social, así como de la consideración de que la puericultura empezaba con el cuidado de la salud de los padres. 


\section{LISTA DE REFERENCIAS}

Andreatta, M. M. y Suárez N. A. (2013). Entre la cocina y el laboratorio: la alimentación científica y las dietistas. Salud i Ciencia, 20(2), 56-60.

Archivos de Pediatría de Uruguay (1940). Anales de la Sociedad de Puericultura de Buenos Aires, vi(35).

Armus, D. y Belmartino, S. (2001). Enfermedades, médicos y cultura higiénica. En A. Cattaruzza (dir.), Nueva historia argentina. Crisis económica, avance del Estado e incertidumbre política, 1930-1943 (pp. 283-330). Buenos Aires: Editorial Sudamericana.

Bayley Bustamente, G. (1941). Proyecto para la solución de un problema higiénico. Envase refrigerante para conservar los biberones. Anales de la Sociedad de Puericultura de Buenos Aires, VII, 46-47.

Bernabeu Mestre, J., Trescastro López, E. y Galiana Sánchez, M. E. (2011). La divulgación radiofónica de la alimentación y la higiene infantil en la España de la segunda república (1933-1935). Salud Pública, 7, 49-60.

Biernat, C. y Ramacciotti, K. (2008). La tutela estatal de la madre y el niño en la Argentina: estructuras administrativas, legislación y cuadros técnicos (1936-1955). História, Ciências, Saúde-Manguinhos, 15(2), 331-351. Dor: https://doi.org/10.1590/ S0104-59702008000200006

Billorou, M. J. (2006). La construcción de la puericultura como campo científico y como política pública en Buenos Aires, 1930-1945. (Tesis inédita de maestría). Facultad de Ciencias Humanas/Universidad Nacional de La Pampa, Santa Rosa.

Bodas de Plata de la Sociedad Uruguaya de Pediatría (1943). Anales de la Sociedad de Puericultura de Buenos Aires, IX(146).

Bogani, G. (1941). El problema del aislamiento en la sala de espera del dispensario. Anales de la Sociedad de Puericultura de Buenos Aires, viI, 23-36.

Briolotti, A. (2016a). La evaluación del desarrollo psicológico en los dispensarios de lactantes de Buenos Aires: medicina y psicología en la Argentina, 1935-1942. História, Ciências, Saúde-Manguinhos, 23(4), 1077-1093. Dor: https://doi.org/10.1590/ S0104-59702016005000022

Briolotti, A. (2016b). Educando a los padres argentinos: un análisis a través de los manuales de puericultura de Aráoz Alfaro y Garrahan. Avances del Cesor. Recuperado de http://web2.rosario-conicet.gov.ar/ojs/index.php/AvancesCesor/index

Briolotti, A. (2018). Pediatría, puericultura y saberes "psi" en el campo del desarrollo infantil: una historia de los usos médicos del conocimiento psicológico en el Río de la Plata, 19301963. (Tesis inédita de doctorado). Universidad Nacional de la Plata, Argentina. 
Boltanski, L. (1969). Puericultura y moral de clase. Barcelona: LAIA.

Buschini, J. (2013). La alimentación como problema científico y objeto de políticas públicas en la Argentina: Pedro Escudero y el Instituto Nacional de la Nutrición, 1928-1946. Apuntes. Revista de Ciencias Sociales, 43(79), 129-156. DoI: https://doi. org/https://doi.org/10.21678/apuntes.79.868

Buschini, J. (2018). La comercialización de la leche de consumo en la ciudad de Buenos Aires y su carácter controversial, 1924-1946. Estudios Sociales del Estado, v(8), 38-60.

Cátedra de Puericultura. Curso para postgraduados (1939). Anales de la Sociedad de Puericultura de Buenos Aires, v, 308-309.

Carreño C. y Cosin, A. (1940). Salas de lactancia. Anales de la Sociedad de Puericultura de Buenos Aires, VI, 113-120.

Cervini, P. R. (1941). La obra de difusión de la puericultura en el interior del país. Conferencia radial. Anales de la Sociedad de Puericultura de Buenos Aires, viI, 59-63.

Colángelo, M. A. (2013). La crianza en disputa: medicalización del cuidado infantil en la Argentina entre 1890 y 1930. (Tesis inédita de doctorado). Facultad de Ciencias Naturales y Museo/Universidad Nacional de la Plata, Buenos Aires.

Creación del Instituto de Puericultura brasilero (1938). Anales de la Sociedad de Puericultura de Buenos Aires, IV(74).

Damianovich, J. (1938). Leche ácida hipergrasosa en el primer trimestre de edad. Anales de la Sociedad de Puericultura de Buenos Aires, IV, 256-262.

Di Liscia, M. S. (2005). Dentro y fuera del hogar. Mujeres, familias y medicalización en Argentina, 1870-1940. Signos Históricos, 7(13), 94-119. Recuperado de http://www. scielo.org.mx/scielo.php?script=sci_arttext\&pid=S1665-44202005000100094

Donzelot, J. (2008). La policía de las familias. Buenos Aires: Ediciones Nueva Visión.

Foucault, M. (2005). La arqueología del saber. Buenos Aires: Editorial Siglo XXI.

Gaing, E. (1938). Nuestra protección municipal a la primera infancia. Su desarrollo y evolución hacia la acción totalitaria. Anales de la Sociedad de Puericultura de Buenos Aires, IV, 11-18.

Gaing, E. (1942). Disontía. Su definición, concepto y porcentaje. Anales de la Sociedad de Puericultura de Buenos Aires, viII, 11-18.

Gavrila, C. (2018). "Sed cual ángeles de piedad, fuertes en vuestra propia debilidad”. La feminización profesional y agenciamiento de las Visitadoras de Higiene Social. 1935-1942. Anuario del Instituto de Historia Argentina, 18(1). DoI: https://doi. org/10.24215/2314-257Xe062

Guiraldes, D. (1944). Sobre la asistencia al recién nacido en nuestra capital. Revista de la Sociedad de Puericultura de Buenos Aires, I, 17-32. 
Gimeno, B. (2018). La lactancia materna. Política e identidad. España: Universidad de Valencia.

Lopes, T. da C. y Maio, M. C. (2018). Puericultura, eugenia e interpretações do Brasil na construção do Departamento Nacional da Criança (1940). Tempo, 24(2), 349368.

Mendilaharzu, J. y Zabala Rodríguez, E. L. (1940). La vitamina C en el lactante. Anales de la Sociedad de Puericultura de Buenos Aires, vi, 109-112.

Mendilaharzu, J., Gentile, M. y Crouzel E. (1942). Hipovitaminosis B en la primera infancia. Anales de la Sociedad de Puericultura de Buenos Aires, viII, 263-270.

Miranda M. (2019). Maternidad y biopolítica en la Argentina. Gregorio Aráoz Alfaro, El Libro de las Madres y la eugenesia, 1870-1955. Passagens. Revista Internacional de História Política e Cultura Jurídica Río de Janeiro, 11(2), 156-176, Dor: https://doi. org/10.15175/1984-2503-201911201

Montagna C. y Burgos H. (1940). Preparación de la mezcla láctea Escudero en las regiones donde se carece de leche fresca. Anales de la Sociedad de Puericultura de Buenos Aires, vi, 98-107.

Murillo, S. (2008). Producción de pobreza y construcción de subjetividad. En Producción de pobreza y desigualdad en América Latina. Bogotá: Siglo del Hombre Editores.

Muñoz, A. de y Escudero, M. (1937) Contribución a la historia de la alimentación del niño. Anales de la Sociedad de Puericultura de Buenos Aires, III, 262-273.

Murín Navarro, J. (1943). La tragedia biológica del interior y la función higiénico-social de los dispensarios en la lucha contra la mortalidad infantil. Anales de la Sociedad de Puericultura de Buenos Aires, Ix, 42-65.

Murtagh, J. y Riviere, C. (1943). La leche cítrica en la alimentación del lactante. Anales de la Sociedad de Puericultura de Buenos Aires, Ix, 24-34.

Nari M. (2005). Políticas de maternidad y maternalismo político, Buenos Aires (1890-1940). Buenos Aires: Biblos.

Primer Congreso Nacional de Puericultura (1940). Anales de la Sociedad de Puericultura de Buenos Aires, vi, 149.

Roman, M. A. (2013). Temas y problemas de salud infantil. Modelos pedagógicos en manuales de Puericultura, Santa Fe, 1915-1925. (Tesis inédita de maestría). Universidad Nacional del Litoral-Facultad de Humanidades y Ciencias, Santa Fe.

Rose, N. (1990). Governing the soul. The shaping of the private self. London: Routledge.

Schweizer, F. (1939) Conferencia inaugural. Anales de la Sociedad de Puericultura de Buenos Aires, IV, 19-23. 
Tiscornia, J. V., Waismann, M. y Crespi, S. (1939). La sífilis pesquisada en el dispensario de lactantes. Anales de la Sociedad de Puericultura de Buenos Aires, v, 271-277.

Virasoro, J. y Roca F. J. (1939). Diarreas. Anales de la Sociedad de Puericultura de Buenos Aires, v, 278-285.

Zwank, A. (1943). La unidad sanitaria en la asistencia de la maternidad y de la infancia. Anales de la Sociedad de Puericultura de Buenos Aires, IX, 1-4. 